Infusionstherapie 1989;16:290-292

\title{
Jahres-Inhalt, Vol. 16, 1989
}

Abstrakts zur 8. Gemeinsamen Jahrestagung der Deutschen Arbeitsgemeinschaft für künstliche Ernährung (DAKE) und der Österreichischen Arbeitsgemeinschaft für klinische Ernährung (AKE), 13.-16. April 1989, Augsburg 4

Simon, J.; Jung, F.; Holbach, T.; Castor, G.;

Jaksche, H.; Kiesewetter, H. (Homburg/Saar)

Intra- und postoperativer Einfluß verschiedener Plasmaersatzmittel auf die Fließfähigkeit des Blutes und den konjunktivalen Sauerstoffpartialdruck bei Mikrobandscheibenoperationen 30

Informationen für die Klinik 39

Mory, M.; Wehner, W. (Karl-Marx-Stadt/DDR)

Die hypokalorische Ernährung in der frühen postoperati-

ven Phase bei Patienten nach kolorektalen Eingriffen... 41 Baumgartl, P.; Pinsker, K. (St. JohanninTirol/Österreich) Anaphylaktischer Schock nach Verabreichung einer fruktosehaltigen Infusionslösung? 45

Balogh, D.; Furtwangler, W.; Hackl, J. M.

(Innsbruck/Österreich), Kahn, J. M. (Bern/Schweiz)

Differenzierte enterale Ernährung in der Postaggressionsphase bei Polytraumatisierten. Vergleich einer hochkalorischen nährstoffdefinierten Diät (WHPC-Diät) mit einer Peptid-Diät 52

Mertes, N.; Winde, G.; Nolte, G.; Keferstein, R.;

Zander, J. (Münster), Pfisterer, M.; Puchstein, Ch.

(Bochum)

Eiweißorientierte peripher-venöse Ernährung nach operativem Trauma 60

Schober, P.H.; Kurz,R.; Musil,H.E. (Graz/Österreich), Jarosch, E. (Innsbruck/Österreich) Streßadaptierte parenterale Aminosäurensubstitution bei operierten Früh- und Neugeborenen 68 Walpot, H.; Franke, R. P.; Burchard, W. G.;

Agternkamp, C; Mutter, F. G.; Mittermayer, Ch.;

Kalff, G. (Aachen)

Untersuchung über die Filtereffektivität herkömmlicher $15-\mu \mathrm{m}$-Filter (DIN 58362) und endständiger 15- $\mu \mathrm{m}$ - und 5- $\mu \mathrm{m}$-Filter. Teil I: Partikelzählung 77 Informationen für die Klinik 81 
Böhler, S.; Wagner, K.; Bässler, K. H. (Mainz)

Metabolismus der L-Thiazolidincarbonsäure-(4) 82

Dommisch, K.; Freesen, P.; Kuhrt, E.; Willroth, P. O.;

Storm, H.; Marin, H. (Schwerin/DDR)

Ernährungsdiagnostische Untersuchungsergebnisse bei

Patienten mit Bronchialkarzinom $\quad 88$

Hebenstreit, G. F.; Fellerer, K.; Twerdy, B.

(Amstetten-Mauer/Österreich), Pfeiffer, P.;

Zadravec, S.; Ferdinand, P. (Graz/Österreich)

L-Tryptophan bei prädeliranten und deliranten Zustands-

bildern 92

Hach-Wunderle, V.; Teixidor, D.; Scharrer, L (Frankfurt), Zumpe, P.; Kühnl, P. (Hamburg)

Anti-A-Titer in einem Faktor-VIII-HS-Konzentrat als Ursache für eine schwere Hämolyse bei

einem Patienten mit erworbenen Hemmkörpern gegen Faktor-VIП: C . 100

Piek, J.; Mahlke, L.; Bock, W. J. (Düsseldorf)

Präoperativer Ernährungszustand neurochirurgischer

Patienten 102

Staedt, t/./ 1·iütt, M.; Herrmann, B.; Seufzer, U.; Leweling, H. (Heidelberg)

Einfluß einer Hämodilution mit 10\%iger Hydroxyäthyl-stärkelösung (MW 200000/0,5) auf die

Fließeigenschaften des Blutes, die arteriellen Blutgase und den konjunktivalen

Sauerstoffpartialdruck bei Patienten mit Hirninfarkt . 107

Buchbesprechungen 113

Mertes, N.; Nolte, G.; Winde, G.; Zander, J. (Münster),

Pfisterer, M.; Puchstein, C. (Herne)

Erfahrungen mit einer neuen Fettemulsion in der opera-

tiven Intensivmedizin 114

Walther, H.; Müller, H.; Aigner, K. R. (Trostberg)

Blutzuckerverlauf und Elektrolyt-Status unter parentera-

ler Ernährung mit einer Komplettlösung nach Dickdarm-

resektion 119

Matkowitz, R-; Hartig, W.; Ritter, R. (Leipzig/DDR), Betzler, H. (Homburg/Saar)

Untersuchungen von Lösungen mit differentem Gehalt an BCAA auf den Proteinstoffwechsel im postoperativen Verlauf- 1. Mitteilung: 15N-Tracer-kinetisches Stoffwech-sel-Tiermodell zur Beurteilung der Wirkung parenteraler Aminosäuren-Zufuhr auf den Stickstoff-Stoffwechsel. . . 125

Informationen für die Klinik 132

Walpot, H.; Franke, R. P.; Burchard, W. G.; Agternkamp, C; Müller, F. G.; Mittermayer, Ch.; Kalff, G. (Aachen)

Untersuchungen über die Filtereffektivität herkömm

licher 15- $\mu \mathrm{m}$-Filter (DIN 58362) - Teil II: Rasterelektro-

nenmikroskopie und Röntgenanalyse 133

Nanoff C; Zwiauer, K.; Widhalm, K. (Wien/Österreich) Follow-up-Untersuchung hochgradig

übergewichtiger Ju-gendlicher 4 Jahre nach stationärer Gewichtsreduktion mit einer

niederkalorischen Protein-Kohlenhydrat-Diät . 141

Jáhres-Inhalt 1989

291 
Jung, F.; Walãhausen, P.; Mrowietz, C; Spitzer, S.;

Kiesewetter, H.; Wenzel, E. (Homburg/Saar)

Hämorheologische, mikro- und makrozirkulatorische Ef-

fekte einer Infusion von $500 \mathrm{ml}$ 6\%iger mittelmolekularer

Hydroxyäthylstärke (Haes 200000/0,5) 148

Butters, M.; Bittner, R.; Metzger, S.; Beger, H. G. (Ulm)

Perioperative Glukoseresorption und hormonelle Reak-

tion nach intraduodenaler Glukoseapplikation $\quad 156$

Dengler, T.; Stacker, U.; Kellner, S.; Fürst, G.

(Baden-Baden)

Chemische und immunochemische Charakterisierung von

Polymeren oder Aggregaten in Human-Serum-Albumin-

Präparationen 160

Lundsgaard-Hansen, P.; Doran, J. E. (Bern),

Blauhut, B. (Linz)

Gibt es einen allgemeingültigen «akzeptablen Min-

destwert» des Hämoglobins? 167

Informationen für die Klinik 175

Zwiauer, K. (Wien)

Bedeutung von Kohlenhydraten in Reduktionsdiäten. . . 176

Kurzmitteilungen

Back, M.; Heim, M. U.; Schleich, I.; Weindler, R.;

Wagner, M.; Mempel, W. (München)

Tbrombozytäre Kreuzprobe mit Capture $\mathrm{P} 囚$ : Klinische

Relevanz 183

Taborski, U. (Bad Nauheim); Mailer, N. (Münster)

Vergleichende Untersuchung über Nebenwirkungen und

Spendersicherheit bei der präparativen Zellgewinnung

mittelsder Zellseparatoren CS 3000 und V50186

Wretlind, A. (Stockholm/Schweden)

Zukunftsperspektiven in der parenteralenErnährung. . . 192

Widhalm, K. (Wien/Österreich)

Der übergewichtige Patient und ein operativer Eingriff . . 199

Eckart, J.; Neeser, G.; Wengert, P.; Adolph, M.

(Augsburg)

Nebenwirkungen und Komplikationen der parenteralen

Ernährung 204

MEDICA-Programm 214

Schmitz, J. E. (Ulm)

Der besondere Ernährungsfall: der septische Patient . . . 216

Puchstein, C, (Bochum), Mertes, N.; Nolle, G. (Münster)

Erfassung des Ernährungszustandes 222

Gïinther, B. (München)

Parenterale Ernährung - periphervenös 230

Wicklmayr, M.; Rett, K.; Baldermann, H.; Dietze, G.; Mehnert, H. (München) 
Künstliche Ernährung bei Diabetikern 233

Thai, P.; Müller, J.M.; Keller, H. W. (Köln)

Führt die parenterale Fettgabe zu einer Beeinflussung der

Immunkompetenz?

237

Daschner, E; Frank, U. (Freiburg)

Probleme der parenteralen Ernährung aus der Sicht des

Krankenhaushygienikers 241

Schmidt, R.; Gollmer, E.; Zunser, R.; Krüger, J.; Ackermann, R. (Köln)

Die Verbreitung der Erythema migrans-Borreliose in

Blutspendern 248

Reissigl, H. (Innsbruck/Österreich)

Bemerkungen zur Arbeit Schmidt et al.

Die Verbreitung der Erythema migrans-Borreliose in

Blutspendern 251

Fauth, U.; Heinrichs, W.; Puénte-Gonzales, I.;

Tzanova, I.; Halmágyi, M. (Mainz)

Verfahren zur Berechnung verschiedener Parameter des

Stoffwechsels von Aminosäurengemischen 253

Heine, W.; Wutzke, K.D.; Radke, M.

(Houston, Tex., USA)

Zur Verminderung der Immunogenität von Milchpro-

teinen durch Desialinisierung 264

Lynen, R.; Baumert, H.; Gallasch, E. (Göttingen)

Stadien der Anti-N-like Immunisierung: ein weiterer

Beitrag zur Anti-N-like-Problematik bei Hämodialyse-

patienten 267

Kurzmitteilungen

Lynen, R.; Sindu, E.; Gallasch, E.; Neumeyer, H.

(Göttingen)

Neuere Untersuchungen über die Häufigkeit von IgG-

Allo-Antikörpern bei Transfusionsempfängern

Seidell, J. C; Hautvast, J. G.A.J.; Deurenberg, P.

(Wageningen/Netherlands)

Ubergewicht: Verteilung des Fettgewebes und Morbidität

-epidemiologischeObservationen. EinÜberblick .... 276

Stock, M.J. (London/UK)

Thermogenese und braunes Fettgewebe: Bedeutung für

menschliche Fettsucht 282

Mitteilungen der Deutschen Gesellschaft für Transfu-

sionsmedizin und Immunhämatologie 285

Autorenverzeichnis $1989 \quad 289$

Jahres-Inhalt $1989 \quad 290$

292

Jahres-Inhalt 1989

Supplement 1 
Reínauer, H. (Düsseldorf)

Insulin als zentraler Stoffwechselregulator unter besonderer Berücksichtigung des Eiweißhaushaltes 3

Jürgens, P. (Hamburg)

Die normale Aminosäurenlösung: Gegenwart und Zu-

kunftsperspektiven 16

Druml, W. (Wien/Österreich)

Die klinische Bedeutung krankheitsadaptierter Amino-

säurenlösungen 26

Schauder, P. (Göttingen)

Einfluß unterschiedlicher Kalorienträger auf den Eiweiß-

haushalt 34

Schmitz, $/ \bullet$ E. (Ulra)

Grenzen und Relation der Aminosäuren- und Energie-

zufuhr 36

Halmágyi, M.; Fauth, U.; Heinrichs, W.; Tzanova, I.

(Mainz)

Aktueller Kalorienwert intravenös infundierter Nähr-

lösungen 44

Keller, H. W.; Mutter, J. M.; Brenner, U.; Walter, M. (Köln),

Kosswig, W. (Bonn)

Effizienz der parenteralen Ernährung 49

Eckart, J.; Neeser, G.; Adolph, M. (Augsburg)

Metabolische Nebenwirkungen der parenteralen Ernäh

rung 55

Supplement 2

Hester, J.; Ventura, G. (Houston, Tex., USA) Gewinnung und Transfusion von Einzelspendern Thrombozytenkonzentrate und ihr klinischer Erfolg .

Kretschmer, V; Söhngen, D.; Göddecke, W.; Pelzer, H.; Prinz, H.; Eckle, R. (Marburg); Kadar, J. G. (Köln) Biokompatibilität und Sicherheit von Zytapheresen ... 10

Borberg, H.; Böhm, R. (Köln)

Die Spendersicherheit in der apparativen Zytapherese . . 21

Urdahl, S. G. (Lakewood, Colo., USA)

COBE Spectra ${ }^{\circledR}-$ Apheresesystem: Ausführung, Versuchs-

protokolle und Ergebnisse $\quad 30$

Weitere Ergebnisse mit dem COBE Spectra ${ }^{\circledR}-S y s t e m$ :

Price, T. H.; Northway, M. M. (Seattle, Wash., USA);

Moore, R. C. (Lakewood, Colo., USA)

Thrombozytengewinnung unter Verwendung des COBE

Spectra ${ }^{\circledR}-$ Systems $\quad 44$

Bertholf, M. F.; Mintz, P. D. (Charlottesville, Va., USA)

Vergleich der Thrombozytapherese mit zwei Zellsepara-

toren unter Verwendung derselben Spender 45

Kevy, S. V.; Jacobson, M. S.; Fosburg, M. T. (Boston, Mass., USA); Moore, R. C. (Lakewood, Colo., USA) Einsatz des COBE Spectra ${ }^{\circledR}-$ Systems (CSS) für Thrombozytapherese und intensiven Plasmaaustausch (IPA).... 46 
Chapman, R. (Denver, Colo., USA); Rank, A.; Holmes, M. (Lakewood, Colo., USA) Simulierte Plasmaaustauschverfahren mit dem COBE Spectra ${ }^{\circledR}$-Apheresesystem bei gesunden Spendern .... 47

Hester, J. P.; Ventura, G. J. (Houston, Tex., USA)

Gewinnung peripherer mononuklearer Blutstammzellen (PBMSC) mit zwei Zellseparatoren mit kontinuierlichem Fluß: Ausbeuteund Reinheit 48

Dutcher, J. P.; Wagner, B.; Ryan, J. (Bronx, NY, USA); Moore, R.; Holmes, M.; Schuyler, B.

(Lakewood, Colo., USA)

Lymphozytopherese für die Gewinnung von Killerzellen und bei Patienten mit chronischer lymphozytischer Leukämie: Vergleich von COBE 2997 mit COBE Spectra ${ }^{\circledR}$. . . 49

Abstrakts zum 23. Kongreß der Deutschen Gesellschaft

für Transfusionsmedizin und Immunhämatologie,

5.-9. September 1989, Kiel 50 